\title{
Biotin dependency due to a defect in biotin transport
}

\author{
Rebecca Mardach, ${ }^{1}$ Janos Zempleni, ${ }^{2}$ Barry Wolf, ${ }^{3}$ Martin J. Cannon, ${ }^{4}$ \\ Michael L. Jennings, ${ }^{5}$ Sally Cress, ${ }^{1}$ Jane Boylan, ${ }^{1}$ Susan Roth,${ }^{1}$ \\ Stephen Cederbaum, ${ }^{1,6}$ and Donald M. Mock ${ }^{2,7}$
}

${ }^{1}$ Regional Metabolic Service, Kaiser Permanente, Los Angeles, California, USA
2 Department of Biochemistry and Molecular Biology, University of Arkansas for Medical Sciences, Little Rock, Arkansas, USA
${ }^{3}$ Department of Pediatrics, Connecticut Children's Medical Center, and the University of Connecticut School of Medicine,
Hartford, Connecticut, USA
${ }^{4}$ Department of Microbiology and Immunology, and
${ }^{5}$ Department of Physiology and Biophysics, University of Arkansas for Medical Sciences, Little Rock, Arkansas, USA
${ }^{6}$ Department of Psychiatry, and Department of Pediatrics, University of California - Los Angeles, Los Angeles, California, USA
${ }^{7}$ Department of Pediatrics, University of Arkansas for Medical Sciences, Little Rock, Arkansas, USA

Address correspondence to: Donald M. Mock, Slot 516, Department of Biochemistry and Molecular Biology,

University of Arkansas for Medical Sciences, 4301 West Markham Street, Little Rock, Arkansas 72205, USA.

Phone: (501) 526-4201; Fax: (501) 603-1146; E-mail: MockDonaldM@uams.edu.

Received for publication April 26, 2001, and accepted in revised form May 14, 2002.

We describe a 3-year-old boy with biotin dependency not caused by biotinidase, holocarboxylase synthetase, or nutritional biotin deficiency. We sought to define the mechanism of his biotin dependency. The child became acutely encephalopathic at age 18 months. Urinary organic acids indicated deficiency of several biotin-dependent carboxylases. Symptoms improved rapidly following biotin supplementation. Serum biotinidase activity and Biotinidase gene sequence were normal. Activities of biotin-dependent carboxylases in PBMCs and cultured skin fibroblasts were normal, excluding biotin holocarboxylase synthetase deficiency. Despite extracellular biotin sufficiency, biotin withdrawal caused recurrent abnormal organic aciduria, indicating intracellular biotin deficiency. Biotin uptake rates into fresh PBMCs from the child and into his PBMCs transformed with Epstein Barr virus were about $10 \%$ of normal fresh and transformed control cells, respectively. For fresh and transformed PBMCs from his parents, biotin uptake rates were consistent with heterozygosity for an autosomal recessive genetic defect. Increased biotin breakdown was ruled out, as were artifacts of biotin supplementation and generalized defects in membrane permeability for biotin. These results provide evidence for a novel genetic defect in biotin transport. This child is the first known with this defect, which should now be included in the identified causes of biotin dependency.

J. Clin. Invest. 109:1617-1623 (2002). doi:10.1172/JCI200213138.

\section{Introduction}

Biotin, a water-soluble vitamin, is the coenzyme for five mammalian carboxylases (1): pyruvate carboxylase (EC 6.4.1.1), propionyl-CoA carboxylase (EC 6.4.1.3), methylcrotonyl-CoA carboxylase (EC 6.4.1.4), and both isoforms of acetyl-CoA carboxylase (EC 6.4.1.2). Biotin holocarboxylase synthetase (EC 6.3.4.10) catalyzes the attachment of biotin to the apocarboxylases by an amide bond to a specific lysine residue-producing holocarboxylases. During normal turnover, biotinylated proteins are proteolytically degraded to biocytin (biotinyl-lysine) and biotinylated oligopeptides that are subsequently cleaved by biotinidase (EC 3.5.1.12), thereby recycling biotin (1).

Both holocarboxylase synthetase deficiency and biotinidase deficiency cause multiple carboxylase deficiency and biotin dependency (1). Severe forms of holocarboxylase synthetase deficiency usually have onset of symptoms during infancy. Clinical features characteristically include feeding problems, acidosis, and an acute progressive encephalopathy. In contrast, biotinidase deficiency characteristically presents after infancy. Features include alopecia, skin rash, developmental delay, hypotonia, seizures, acidemia, aciduria, hearing problems, and vision problems. Biotinidase deficiency may be clinically indistinguishable from mild forms of holocarboxylase synthetase deficiency (2). Both disorders typically respond to biotin therapy. Both nutritional biotin deficiency and inherited enzymatic deficiencies of the biotin-dependent carboxylases cause abnormally increased urinary excretion of characteristic organic acids $(1,3,4)$. These compounds include the following: (a) 3-methylcrotonylglycine and 3-hydroxyisovalerate (3HIA) reflecting methylcrotonyl-CoA carboxylase deficiency; (b) 3-hydroxypropionate (3HPA), propionylglycine, and methylcitrate reflecting propionyl-CoA carboxylase deficiency, and (c) lactate, which likely reflects pyruvate carboxylase deficiency.

We now describe a child with biotin dependency who does not have an isolated carboxylase deficiency, holocarboxylase synthetase deficiency, or biotinidase deficiency. We present evidence that his biotin dependency is the result of a defect in biotin transport. 
Case description. The proband is the only child of nonconsanguineous Asian parents. He was born prematurely at 26 weeks of gestation but suffered no obvious long-term deleterious effects from his premature birth. During infancy, he consumed commercial infant formulas containing amounts of biotin that met or exceeded the recommended intake for his age. As an older infant and toddler, he consumed a mixed general diet. Growth and development were normal without abnormal hair loss or skin rash until 18 months of age and the onset of an acute illness consistent with viral gastroenteritis. After 3 days of irritability and poor fluid intake, he deteriorated neurologically. He became progressively lethargic and finally completely obtunded. Venous blood $\mathrm{pH}$ was 6.97 , and bicarbonate concentration was less than $5 \mathrm{mEq} / 1$ (normal 18-30 $\mathrm{mEq} / \mathrm{l})$. Plasma lactate concentration was $14 \mathrm{~mol} / \mathrm{l}$ (normal 0.7-2.0 mol/1). Blood ammonia concentration was $109 \mathrm{mmol} / \mathrm{l}$ (normal 10-50 mmol/l). Plasma glucose concentration was $1.0 \mathrm{mmol} / 1$ (normal 3.3-5.5 $\mathrm{mmol} / \mathrm{l})$. These laboratory findings are consistent with the profound hepatocellular dysfunction characteristic of many inborn errors of metabolism.

The pattern of urinary organic acid excretion determined by the Children's Hospital of Los Angeles Metabolic Laboratory (Los Angeles, California, USA) was diagnostic of multiple carboxylase deficiency (Table 1). Biotin supplementation was initiated at $10 \mathrm{mg} / \mathrm{d}$ (41 $\mu \mathrm{mol} / \mathrm{d}$ ) orally. Carnitine therapy $(900 \mathrm{mg} / \mathrm{d}=5.6$ $\mathrm{mmol} / \mathrm{d}$ ) was initiated because secondary carnitine deficiency usually occurs in the biotin-dependent carboxylase deficiencies (5). Biotin supplementation was continued as an outpatient, whereas carnitine was discontinued once the plasma concentration of carnitine returned to normal. Three months after the encephalopathic episode, the child had not completely regained his vocabulary or independent ambulation. Over the second and third year of life, he gradually regained normal neuropsychological and motor developmental milestones. He is currently developmentally normal.

\section{Methods}

Study design. To determine whether the child exhibited persistent biotin dependency, two 6-week trials of discontinuing biotin supplementation were undertaken. Urinary excretion of 3HIA was monitored on a weekly basis using a standard organic acid method (6). The child was closely monitored by his parents for any signs or symptoms resembling his acute illness.

Organic acid analyses. The initial organic acid analyses were performed at Children's Hospital of Los Angeles using a silica gel technique that extracts variable proportions of most hydrophilic compounds (standard method); extraction is followed by derivatization and identification and quantitation by standard gas chromatography/mass spectroscopy techniques (6). Final quantitation of urinary 3HIA during biotin withdrawal used a technique optimized for 3HIA quantitation; deuterated and unlabeled 3HIA served as internal and external standards, respectively (optimized method) (7). The same technique was used to measure 3HPA; deuterated 3HIA and unlabeled 3HPA served as the internal and external standards, respectively. Normal ranges for both 3HIA and 3HPA were calculated as plus or minus 2 SD from a population of 20 normal adults consuming a mixed general diet. These normal ranges agreed with those reported for 3HIA by the Children's Hospital of Los Angeles and for 3HPA as reported by Kalloghlian et al. (8).

Biotin, biocytin, and biotin catabolites. Biotin, biocytin, and the two principal biotin catabolites, bisnorbiotin and biotin sulfoxide, were separated by reverse-phase HPLC (9). Individual HPLC fractions were then quantitated against authentic standards, as described previously, using an avidin-binding assay (9).

Enzyme activities. Biotinidase activity was measured by the method of Wolf et al. (10). The activities of three biotin-dependent carboxylases were determined in extracts of both PBMCs and cultured skin fibroblasts by the Biochemical Genetics Service Laboratory at the University of California, San Diego (San Diego, California, USA), using ${ }^{14} \mathrm{CO}_{2}$ incorporation assays $(11,12)$. Assays were conducted on cells cultured in Eagle's minimal essential media containing $10 \%$ FCS; the media biotin content is typically $6 \mathrm{nmol} / 1$. Enzymatic assays were conducted in buffers containing no added biotin.

Isolation of PBMCs and erythrocytes. PBMCs, which are primarily lymphocytes (13), and erythrocytes were isolated from heparinized venous blood by gradient centrifugation using Histopaque (Sigma-Aldrich, St. Louis, Missouri, USA) as described previously (14). After shipment of blood samples of the proband and his parents to the University of Arkansas for Medical Sciences (Little Rock, Arkansas, USA), lymphocyte viability remained greater than $97 \%$ by Trypan blue exclusion (14).

Biotin and pantothenic acid transport in PBMCs and erythrocytes. Transport of biotin and pantothenic acid were measured using $\left[{ }^{3} \mathrm{H}\right]$ biotin and $\left[{ }^{3} \mathrm{H}\right]$ pantothenic acid, respectively, as described previously $(14,15)$.

Sequencing the biotinidase gene. DNA was isolated from peripheral blood. The exonic and adjacent intronic regions of the gene were directly sequenced as described previously (2).

\section{Table 1}

Diagnostic organic aciduria prior to biotin therapy

3-Hydroxyisovaleric acid
3-Methylcrotonylglycine
Tiglylglycine
3-Hydroxypropionic acid
Propionylglycine
Methylcitric acid
Lactic acid
Pyruvic acid

\begin{tabular}{cc}
$\begin{array}{c}\text { Child } \\
(\mathrm{mmol} / \mathrm{mol} \text { creatinine })\end{array}$ & Normal range \\
3,970 & $2-52$ \\
1,075 & $<1$ \\
504 & $<3$ \\
2,233 & $2-28$ \\
703 & $<1$ \\
309 & $<13$ \\
47,630 & $7-94$ \\
428 & $3-43$ \\
\hline
\end{tabular}

AFrom the Los Angeles Children's Hospital Metabolic Laboratory. 
Table 2

Enzyme activities

\begin{tabular}{lccr}
\hline Enzyme & Tissue source & Child's value & Normal range \\
Methylcrotonyl-CoA carboxylase & Fibroblasts & $104^{\mathrm{A}}$ & $17-250^{\mathrm{A}}$ \\
& Leukocytes & $67^{\mathrm{A}}$ & $62-288^{\mathrm{A}}$ \\
Propionyl-CoA carboxylase & Fibroblasts & $299^{\mathrm{A}}$ & $128-537^{\mathrm{A}}$ \\
& Leukocytes & $96^{\mathrm{A}}$ & $160-447^{\mathrm{A}}$ \\
Pyruvate carboxylase & Fibroblasts & $298^{\mathrm{A}}$ & $93-362^{\mathrm{A}}$ \\
& Leukocytes & $19.8^{\mathrm{A}}$ & $6.5-40.7^{\mathrm{A}}$ \\
Biotinidase & Serum & $7.02^{\mathrm{B}}$ & $4.0-11^{\mathrm{B}}$
\end{tabular}

${ }^{A} p m o l\left(\min \times \mathrm{mg}\right.$ protein); normal range from Biochemical Genetics, University of California - San Diego. ${ }^{\mathrm{B}} \mathrm{nmol}(\mathrm{min} \times \mathrm{ml}$ serum); normal range (1).

Sequencing of the sodium-dependent multivitamin transporter gene. As described previously (15), total RNA was isolated from peripheral blood, and cDNA for the sodium-dependent multivitamin transporter (SMVT) gene was isolated by RT-PCR, using customized oligonucleotide primers. The cDNA produced was cloned and sequenced as described previously (15). For each fragment, one or two clones were sequenced. Each fragment was sequenced at least one time from each direction.

Epstein Barr virus-transformation of PBMCs. PBMCs were immortalized using the EBV method (16). Normal control individuals providing cells for transformation were not the same individuals who provided blood for measurement of biotin uptake in quiescent PBMCs.

\section{Results}

Biotin withdrawal. We assessed whether continued biotin therapy was required by withdrawing biotin therapy on two occasions as described in Methods. No overt signs or symptoms occurred during either trial. Biotin supplementation was reinstituted at $10 \mathrm{mg} / \mathrm{d}$ (41 $\mu \mathrm{mol} / \mathrm{d})$ when abnormally increased 3HIA excretion was detected by the relatively insensitive standard method (6). Final quantitation by the optimized method for 3HIA quantitation detected persisting abnormal 3HIA excretion even before biotin withdrawal (Figure 1). By the third week of withdrawal, 3HIA excretion had increased tenfold. During the second withdrawal trial, the 3HIA excretion pattern was similar to the first (Figure 1). Both trials provided evidence of persisting and progressive decreases in methylcrotonyl-CoA carboxylase activity.

With biotin therapy, 3HPA excretion was initially normal. By the third week of withdrawal, 3HPA excretion had increased to greater than twice the upper limit of normal, providing evidence of decreased propionylCoA carboxylase activity. A similar pattern was observed during the second trial of biotin withdrawal. The urinary excretion rates of 3-methylcrotonyl glycine, isovaleryl glycine, and methylcitrate were normal during both withdrawal trials.

Enzyme activity. Activities of the three intramitochondrial biotin-dependent carboxylases were normal in both leukocytes and fibroblasts from the child, thus ruling out isolated deficiencies of these enzymes as causes for his biotin dependency (Table 2; normal range from Biochemical Genetics, University of California - San Diego, San Diego, California, USA). Normal activity of these carboxylases in leukocytes also excludes holocarboxylase synthetase deficiency.

We investigated the possibility of a common variant biotinidase deficiency or of an unusual variant in biotinidase leading to normal maximal activity in the presence of saturating substrate (biocytin) but reduced activity at normal substrate concentrations. Biotinidase activity in the proband's serum was normal (Table 2), thereby indicating that the enzyme has normal catalytic activity and is secreted normally from the tissues. His biotinidase gene was completely sequenced; no alterations in the coding sequence or in the adjacent intronic sequences were found. Moreover, the ratio of biocytin to biotin in urine was not increased during either biotin therapy or withdrawal (Figure 2a), indicating that biotinidase was functioning normally in vivo.

We assessed whether biotin therapy had returned plasma concentrations of biotin at least to normal. The biotin concentration in a blood sample obtained 48 hours after the last biotin dose was 22,500 pmol/1; the normal range is 140 to $365 \mathrm{pmol} / \mathrm{l}$ (17). Moreover, urinary excretion of biotin was substantially elevated

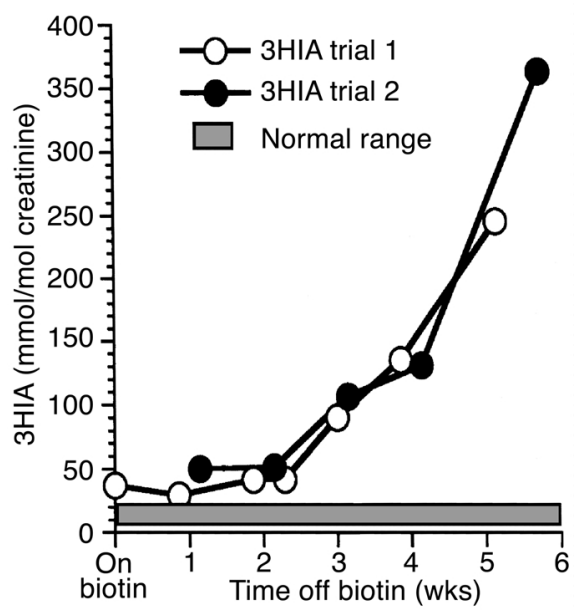

Figure 1

Urinary excretion of $3 \mathrm{HIA}$ during biotin withdrawal. White circles denote trial number 1 , and black circles denote trial number 2 . 
a

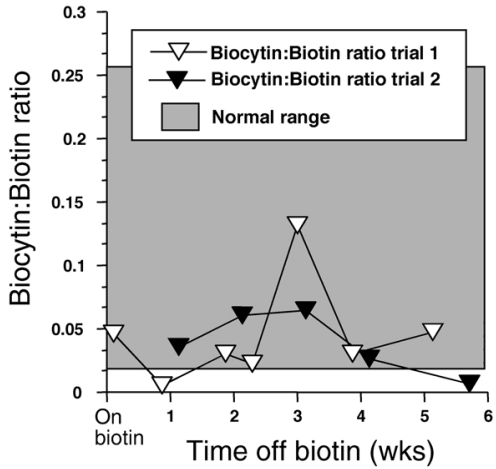

b

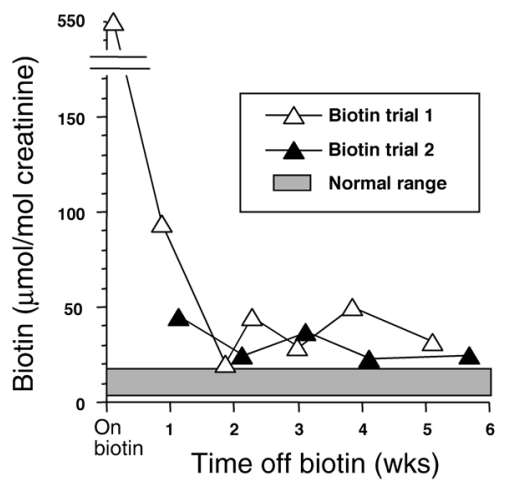

Figure 2

Urinary excretion of biotin and biocytin during biotin withdrawal. (a) Ratio of biocytin to biotin and (b) biotin.

during biotin therapy and decreased strikingly during biotin withdrawal (Figure $2 b$ ).

The striking contrast between apparent extracellular biotin excess and apparent intracellular biotin deficiency suggested the possibility of a genetic defect in biotin transport into tissues. On three occasions, biotin uptake was determined in freshly isolated PBMCs from the proband and his parents. On each occasion, the child had been on biotin therapy $(10 \mathrm{mg} / \mathrm{d}=41 \mu \mathrm{mol} / \mathrm{d})$ for at least 1 month. In an effort to minimize artifacts arising from large plasma concentrations of biotin, biotin therapy was discontinued 48 hours before obtaining blood. The child's mean biotin transport rate was approximately $10 \%$ of the mean for the adult controls (Table 3). To control for a potential age effect, biotin transport was measured in the cord blood of healthy newborns; his biotin transport rate was substantially less than any of the newborns (Table 3). Biotin transport for the father and the mother were less than the normal adult controls; their values averaged 35\% and $65 \%$, respectively, of the normal adult mean (Table 3 ). These values are consistent with (but not direct proof of) heterozygosity for an autosomal recessive genetic defect in biotin transport.

We evaluated the possibility that the child's low activity of the biotin transporter could be caused by an artifactual dilution of specific activity of $\left[{ }^{3} \mathrm{H}\right]$ biotin. However, the carryover of endogenous biotin into the transport assay is only $0.02 \%$; thus is the contribution from an endogenous biotin concentration of 22,500 pmol/l would be less than $5 \mathrm{pmol} / \mathrm{l}$. We further confirmed the absence of such an effect by demonstrating that the in vitro addition of approximately 20,000 $\mathrm{pmol} / \mathrm{l}$ of biotin to blood samples of three normal adults did not have a significant effect on biotin transport activity.

We investigated the possibility that chronic in vivo biotin therapy produced a physiologic downregulation of the biotin transporter in the child. Five normal adults consumed $1 \mathrm{mg}$ of biotin daily for 7 days, producing a mean biotin plasma level of $34,600 \pm 8,000 \mathrm{pmol} / \mathrm{l}$ in blood samples drawn 1-3 hours after the last biotin dose. In these individuals, mean biotin transport activity was not significantly different before biotin supplementation $\left(874 \pm 135 \mathrm{amol} / 10^{6}\right.$ cells $\left.\times 30 \mathrm{~min}\right) \mathrm{com}-$ pared with after biotin supplementation $(687 \pm 243$ amol $/ 10^{6}$ cells $\times 30 \mathrm{~min}$ ). $P$ values were more than 0.3 as determined by paired $t$ test.

We investigated whether the putative genetic biotin transport defect would be expressed in the family's EBV-transformed PBMCs. The child's rate was $10 \%$ of the mean biotin transport of the normal controls (Table 3). Biotin transport for the father and the mother were less than the normal adult controls; their values averaged $19 \%$ and $20 \%$, respectively, of the normal adult mean (Table 3). These observations provide further evidence for genetic defect in biotin transport.

The SMVT transports pantothenic acid, lipoic acid, and biotin. As such, SMVT was a candidate molecule for the defective transporter in this child. The function of SMVT and its genetic sequence were investigated in the proband as follows. His pantothenic acid transport rate (mean $\pm 1 \mathrm{SD}$ ) was $13 \pm 3 \mathrm{fmol} /\left(10^{6}\right.$ cells $\times 15 \mathrm{~min} ; n=3)$. The mean pantothenic acid transport rate $( \pm 1 \mathrm{SD})$ for the five normal adults was $17 \pm 4 \mathrm{fmol} /\left(10^{6}\right.$ cells $\left.\times 15 \mathrm{~min}\right)$; the range was 12.9-21.8. Thus, the child's pantothenic acid transport rate was normal. We also sequenced his SMVT gene. The proband's SMVT gene sequence was $100 \%$ identical with the published sequence (18).

Although unlikely to explain all the laboratory findings presented above, we investigated two additional possible mechanisms for the putative genetic defect: a greatly increased rate of the biotransformation of biotin to one of its inactive catabolites and an alteration in the inherent permeability of the proband's plasma membranes to biotin. The two primary biotin catabolites (bisnorbiotin and biotin sulfoxide) were quantitated in urine samples collected before and during the withdrawal trials. Urinary excretion of bisnorbiotin and biotin sulfoxide were increased appropriately during biotin therapy and decreased appropriately during biotin withdrawal (data not shown). These urine samples were also screened for unknown avidin-binding substances that could indicate novel biotin catabolites; none were detected. To measure membrane permeability to biotin in the absence of the transporter, we measured the uptake of biotin in erythrocytes from the proband, his parents, and six normal controls. Biotin 
Table 3

Biotin transport rates in quiescent and EBV-transformed $\mathrm{PBMCs}^{\mathrm{A}}$

\begin{tabular}{|c|c|c|c|}
\hline Quiescent & Mean $^{B}$ & SD & Range \\
\hline Proband & 75 & 65 & $33-150$ \\
\hline Father & 254 & 30 & $219-272$ \\
\hline Mother & 478 & 162 & $291-575$ \\
\hline Adult controls ${ }^{C}$ & 728 & 135 & $724-987$ \\
\hline Newborn controls ${ }^{C}$ & 414 & 75 & $332-491$ \\
\hline EBV-transformed & Mean ${ }^{B}$ & SD & Range \\
\hline Proband & 649 & 156 & $532-826$ \\
\hline Father & 1,258 & 417 & $791-1,594$ \\
\hline Mother & 1,355 & 1,355 & $1,130-1,521$ \\
\hline Adult controls ${ }^{\mathrm{D}}$ & 6,752 & 6,688 & $2,038-16,643$ \\
\hline
\end{tabular}

Aamol $/\left(10^{6}\right.$ cells $\left.\times 30 \mathrm{~min}\right)$. ${ }^{\text {BMean }}$ of three separate determinations for proband and parents. Each determination was done in triplicate; the coefficient of variation among replicates (the analytical variability) was consistently $<10 \%$. $\mathrm{C}_{n}=5 ; \mathrm{D}_{n}=6$

uptake was $2.0,2.0$, and $2.3 \mathrm{amol} /\left(10^{6} \mathrm{cells} \times 30 \mathrm{~min}\right)$ for the proband and his parents: controls averaged 2.7 with a range of 2.1-3.2. This minimal uptake may represent a slow nonspecific binding in view of the observations that the "uptake" rate decreased noticeably after the first 15 minutes and that the rate is equivalent to uptake of about one molecule of biotin per erythrocyte per 30 minutes. We infer that plasma membranes, in general, and erythrocyte plasma membranes, in particular, are highly impermeable to biotin.

\section{Discussion}

The studies presented here indicate biotin dependency in this child and provide evidence that the cause of his dependency is an inherited defect in biotin transport. On the basis of his clinical history, we conclude that he was in a state of precarious biotin homeostasis and that a routine febrile illness was sufficient to precipitate a near-fatal episode of biotin deficiency. The activities of several biotin-dependent carboxylases were profoundly decreased at presentation as judged by his increased urinary excretion of diagnostic organic acids. Because he exhibited biochemical biotin dependency 8 months and 13 months after his initial presentation, his biotin dependency is a persistent condition and is consistent with a genetic etiology. The strongest evidence in favor of a genetically determined biotin-transport defect is the demonstration of impaired biotin transport in both fresh and EBV-transformed PBMCs from this child as compared with the appropriate controls. A genetic basis for this biotin transport defect is further supported by the observation that biotin transport rates in both fresh and EBV-transformed PBMCs from both parents were less than those of the normal adult controls.

Our studies provided evidence that this child did not have either of the two established genetic causes of multiple carboxylase deficiency: biotinidase deficiency and holocarboxylase synthetase deficiency. Normal serum biotinidase activity and gene sequence excluded biotinidase deficiency. The normal carboxylase activities in extracts of peripheral blood leukocytes and in fibroblasts cultured in medium with biotin concentrations of approximately $6 \mathrm{nmol} / \mathrm{l}$ indicate that the child does not have holocarboxylase synthetase deficiency. Isolated genetic deficiencies of methylcrotonyl-CoA carboxylase, propionyl-CoA carboxylase, and pyruvate carboxylase were also excluded.

The finding of normal carboxylase activities in extracts of leukocytes and of cultured fibroblasts would seem to conflict with our central hypothesis that the intracellular environment is biotin depleted as the consequence of a biotin transport defect. However, we propose that intracellular deficiency occurred during normal biotin intake and began to reoccur during biotin withdrawal. We further propose that supplementation with pharmacologic amounts of biotin in vitro and in vivo at least partially corrects this intracellular deficiency.

The observation that the absolute rate of biotin uptake in the child's EBV-transformed PBMCs is tenfold greater than the rate in his quiescent, untransformed PBMCs is noteworthy. Stimulation of PBMCs with mitogens increases biotin transport two- to tenfold (19). The increased proliferation associated with immortalization recapitulates the increased cell proliferation associated with mitogen stimulation. Thus, an increase in biotin transport rate in immortalized cells is to be expected for both normal individuals and the proband. However, the reduction in biotin transport relative to EBV-transformed cells from normal individuals was preserved in the proband and, in fact, accentuated in the parents by EBV transformation.

Biotin is taken up actively from the lumen of the gastrointestinal tract by a structurally specific, $\mathrm{Na}^{+}-$ coupled transport process (20) and is transported in plasma primarily free in solution (3). Biotin is also transported into tissues by a structurally specific, $\mathrm{Na}^{+}$-coupled process (21). Biotin is catabolized primarily by $\beta$-oxidation of the valeric acid side chain to bisnorbiotin and related catabolites (22) and by sulfur oxidation to biotin sulfoxide and related catabolites (3). Biotin and biotin catabolites are excreted primarily by the kidney. During deficiency, renal excretion of biotin and its catabolites are downregulated, conserving total body biotin (4). Following administration of pharmacologic amounts of biotin, renal excretion of both biotin and its catabolites increase substantially (23).

This child exhibited deficiency of multiple carboxylases at presentation. He exhibited deficiency of methylcrotonyl-CoA carboxylase and propionyl-CoA carboxylase during each trial of biotin withdrawal. These observations suggest that the intracellular environment was biotin deficient and is consistent with a defect of biotin transport from the plasma into somatic cells. Although the somatic cells tested in this study were fresh and EBV-transformed PBMCs, the profound dysfunction of the child's intermediary metabolism and CNS suggest that the defect in biotin transport is more generalized. Possibilities include abnormalities in 
hepatocyte biotin uptake, renal biotin salvage, intestinal biotin absorption, biotin transport across the blood-brain barrier, biotin transport into neurons, or a combination of these defects.

The observation that the child's plasma concentration of biotin was $22,500 \mathrm{pmol} / \mathrm{ml} 48$ hours after discontinuing biotin therapy does not argue for or against an intestinal biotin transport defect. Whether or not the transporter defect is expressed in the intestine, increased plasma concentrations can be produced by simple diffusion; the rapid half-life of disappearance of biotin from plasma (approximately 20 minutes) observed in swine at physiologic doses (24) and in normal adult volunteers at pharmacologic biotin doses (D.M. Mock, unpublished observations) is consistent with greatly increased plasma concentrations of biotin decreasing rapidly to values that are only about 20 times normal.

Studies of biotin transport into erythrocytes provide the evidence that biotin cannot cross the plasma membrane at meaningful rates in the absence of a transporter. Studies using isolated hepatocytes and brush-border membrane preparations from animals and humans indicate that biotin transport from plasma into liver parenchyma is $\mathrm{Na}^{+}$-coupled, electroneutral, structurally specific, saturable, and requires energy $(21,25-30)$. These characteristics are consistent with one (or more) biotin transporters. Reported $K_{\mathrm{T}}$ values are approximately $100 \mu \mathrm{mol} / \mathrm{l}$ and are thus substantially above the normal plasma concentration of biotin $(0.5 \mathrm{nmol} / \mathrm{l})$. A similar system operates to absorb biotin from the intestinal lumen (20). Biotin reclamation by the kidney is $\mathrm{Na}^{+}$-coupled, structurally specific, saturable, and requires energy (31-33), suggesting the existence of one or more renal biotin transporters. The observed $K_{\mathrm{T}}$ is approximately $300 \mu \mathrm{mol} / 1$. These reported values are substantially above the concentration of biotin in plasma and in the glomerular filtrate (probably about $0.5 \mathrm{nmol} / \mathrm{l}$ ), raising the question of the physiologic relevance of these studies. At such concentrations, transport is likely mediated by the SMVT as described above, and conclusions reached may not be directly applicable to biotin transport at physiological concentrations of biotin.

Two candidate biotin transporters have been described: a multivitamin transporter present in many tissues and a biotin transporter identified in human PBMCs. In 1997, Prasad and coworkers reported a pantothenic acid transporter present in human placental choriocarcinoma cells (34). Transport of two additional vitamins - biotin and lipoic acid - plus the demonstration of mutual pairwise inhibition suggested that this transporter can transport all three vitamins. This SMVT has been cloned from two human cell lines: the JAR choriocarcinoma (35) and the Caco-2 intestinal cell line (18). Expression of the SMVT in Xenopus lavis oocytes revealed properties similar to those found in vivo. The SMVT gene is widely expressed in human tissues. This SMVT protein is theoretically a candidate for the defective biotin transporter in this child. However, the reported values for $K_{\mathrm{T}}$ (approximately $10 \mu \mathrm{mol} / \mathrm{l}$ ) are much greater than the $K_{\mathrm{T}}$ of the lymphocyte transporter. Moreover, transport of pantothenic acid into fresh PBMCs of the child was normal, and his SMVT cDNA sequence was normal. Finally, mutual inhibition of transport between biotin and pantothenic acid implies that these molecules at least share some aspect of the transporter binding site. To attribute the results of the studies reported here to a genetic defect in the SMVT, one would have to postulate a genetic but posttranslational modification of the SMVT protein that virtually eliminated biotin transport without significantly affecting pantothenic acid transport, despite the shared binding site.

The biotin transporter identified in PBMCs is $\mathrm{Na}^{+}-$ coupled, saturable, structurally specific, and requires energy. The apparent $K_{\mathrm{T}}$ is $2.6 \mathrm{nmol} / \mathrm{l}$, a value closer to normal plasma concentrations of biotin (14). In contrast to the SMVT, biotin transport in PBMCs is not easily inhibited by pantothenic acid (19). We contend that such differences likely indicate that the lymphocyte biotin transporter is a different protein from the SMVT and that the lymphocyte biotin transporter is a better candidate for the defective biotin transporter.

More than 20 years ago, patients with multiple carboxylase deficiency, decreased plasma and urinary biotin, and/or blunted plasma biotin concentrations in response to orally administered biotin were postulated to suffer from a genetic defect in intestinal biotin transport $(36,37)$. Although these children ultimately proved to have biotinidase deficiency (38), the original hypothesis that a biotin transporter defect would present as biotin dependency seems to be correct based on the observations in the child discussed here. A defect in biotin transport should now be included in the differential diagnosis of biotin dependency.

\section{Acknowledgments}

This work was supported by NIH grants DK-36823 (to D.M. Mock) and GM-26861 (to M.L. Jennings); NIH grant DK-3384 and the Safra Research Fund (to B. Wolf); and United States Public Health Service grants HD-06576, HD-36415 (to S. Cederbaum), and HD-04612 (to the University of California - Los Angeles, Mental Retardation Research Center). The initial diagnosis of multiple carboxylase deficiency was made at Children's Hospital of Los Angeles by Julian Williams. The Regional Metabolic Laboratory at Kaiser Permanente under the Direction of Mehdi Jamehdor and Tullio Giudci performed initial urine organic acid analyses. Darrell Thorne provided able administrative support at the University of California - Los Angeles. Shawna Owens, Cecil Bogy, Melain Raguseo, and Teresa Evans performed measurements of biotin transport, urinary biotin, biocytin, biotin catabolites, 3HPA, and 3HIA. 
1. Wolf, B. 2001. Disorders of biotin metabolism. In The Metabolic and Molecular Basis of Inherited Disease. C.R. Scriver, A.L. Beaudet, W.S. Sly, and D Valle, editors. McGraw-Hill Inc. New York, New York, USA. 3151-3177.

2. Pomponio, R.J., et al. 1997. Mutations in the human biotinidase gene that cause profound biotinidase deficiency in symptomatic children: molecular, biochemical and clinical analysis. Pediatr. Res. 42:840-848.

3. Mock, D.M. 1996. Biotin. In Present knowledge in nutrition. E.E. Ziegler, and L.J. Filer, Jr., editors. USA International Life Sciences Institutes Nutrition Foundation. Washington, DC, USA. 220-235.

4. Mock, D.M. 1999. Biotin status: which are valid indicators and how do we know? J. Nutr. 129(Suppl):498S-503S.

5. Sweetman, L., and Williams, J.C. 1995. Branched chain organic acidurias. In The metabolic and molecular bases of inherited disease. C.R. Scriver, A.L. Beaudet, W.S. Sly, and D. Valle, editors. McGraw-Hill Inc. New York, New York, USA. 1387-1422.

6. Sweetman, L. 1984. Qualitative and quantitative analysis of organic acids in physiologic fluids for diagnosis of the organic acidurias. In Abnor malities of amino acid metabolism in clinical medicine. W.L. Nyhan, editor. Appleton-Century-Crofts. Norwalk, Connecticut, USA. 419-453.

7. Mock, D.M., Jackson, H., Lankford, G.L., Mock, N.I., and Weintraub, S.T 1989. Quantitation of urinary 3-hydroxyisovaleric acid using deuterated 3-hydroxyisovaleric acid as internal standard. Biomed. Environ. Mass Spectrom. 18:652-656.

8. Kalloghlian, A. 1992. A patient with propionic acidemia managed with continuous insulin infusion and total parenteral nutrition. J. Child. Neurol. 7(Suppl.):S88.

9. Mock, D.M. 1997. Determinations of biotin in biological fluids. In Methods in enzymology. D.B. McCormick, J.W. Suttie, and C. Wagner, editors. Academic Press. New York, New York, USA. 265-275.

10. Wolf, B., Grier, R.E., Allen, R.J., Goodman, S.I., and Kien, C.L. 1983. Biotinidase deficiency: an enzymatic defect in late-onset multiple carboxylase deficiency. Clin. Chim. Acta. 131:273-281.

11. Weyler, W., Sweetman, L., Maggio, D.C., and Nyhan, W.L. 1977. Deficiency of propionyl-CoA carboxylase and methylcrotonyl-CoA carboxylase in a patient with methylcrotonylglycinuria. Clin. Chim. Acta. 76:321-328

12. Utter, M.F., and Keech, D.B. 1963. Pyruvate carboxylase: nature of the reaction. J. Biol. Chem. 283:2603-2608.

13. Ratnoff, O.D. 1988. Blood. In Physiology. R.M. Berne, and M.N. Levy, editors. C. V. Mosby Co. St. Louis, Missouri, USA. 357-370.

14. Zempleni, J., and Mock, D.M. 1998. Uptake and metabolism of biotin by human peripheral blood mononuclear cells. Am. J. Physiol. 275:C382-C388.

15. Zempleni, J., Stanley, J.S., and Mock, D.M. 2001. Proliferation of peripheral blood mononuclear cells causes increased expression of the sodiumdependent multivitamin transporter gene and increased uptake of pantothenic acid. J. Nutr. Biochem. 12:465-473.

16. Coico, R., et al., editors. 1991. Current protocols in immunology. John Wiley $\&$ Sons. New York, New York, USA. 7.22.21-7.22.23.

17. Mock, D.M.M., and Nell, I. 2002. Lymphocyte propionyl-CoA carboxylase activity is an early and sensitive indicator of biotin deficiency in rats, but urinary excretion of 3-hydroxyisopropionic acid is not. J. Nutr. In press.

18. Wang, $\mathrm{H}$., et al. 1999. Human placental $\mathrm{Na}^{+-d e p e n d e n t ~ m u l t i v i t a m i n ~}$ transporter. J. Biol. Chem. 274:14875-14883.

19. Zempleni, J., and Mock, D.M. 1999. Mitogen-induced proliferation increases biotin uptake into human peripheral blood mononuclear cells. Am. J. Physiol. 276:C1079-C1084.
20. Said, H.M. 1999. Cellular uptake of biotin: mechanisms and regulation. J. Nutr. 129(Suppl.):490S-493S.

21. McCormick, D.B., and Zhang, Z. 1993. Cellular assimilation of watersoluble vitamins in the mammal: riboflavin, B6, biotin, and C. Proc. Soc. Exp. Biol. Med. 202:265-270.

22. Zempleni, J., and Mock, D.M. 1999. Advanced analysis of biotin metabolites in body fluids allows a more accurate measurement of biotin bioavailability and metabolism in humans. J. Nutr. 129(Suppl.):494S-497S

23. Mock, D.M., and Heird, G.M. 1997. Urinary biotin analogs increase in humans during chronic supplementation: the analogs are biotin metabolites. Am. J. Physiol. 272:83-87.

24. Mock, D.M., Wang, K.S., and Kearns, G.L. 2001. The clearance and metabolism of biotin administered intravenously to pigs at tracer and physiologic amounts is much more rapid than previously appreciated. J. Nutr. 131:1271-1278.

25. Bowers-Komro, D.M., and McCormick, D.B. 1985. Biotin uptake by isolated rat liver hepatocytes. In Biotin. K. Dakshinamurti and H.N. Bhagavan, editors. New York Academy of Sciences. New York, New York, USA. 350-358.

26. Said, H.M., Korchid, S., and Horne, D.W. 1990. Transport of biotin in basolateral membrane vesicles of rat liver. Am. J. Physiol. 259:G865-G872.

27. Weiner, D., and Wolf, B. 1991. Biotin uptake, utilization, and efflux in normal and biotin-deficient rat hepatocytes. Biochem. Med. Metab. Biol. 46:344-363.

28. Weiner, D., and Wolf, B. 1990. Biotin uptake in cultured hepatocytes from normal and biotin-deficient rats. Biochem. Med. Metab. Biol. 44:271-281.

29. Said, H.M., Hoefs, J., Mohammadkhani, R., and Horne, D. 1992. Biotin transport in human liver basolateral membrane vesicles: a carrier-mediated, $\mathrm{Na}+$ gradient-dependent process. Gastroenterology. 10:2120-2125.

30. Said, H.M., Ma, T.Y., and Kamanna, V.S. 1994. Uptake of biotin by human hepatoma cell line, Hep G(2): a carrier-mediated process similar to that of normal liver. J. Cell Physiol. 161:483-489.

31. Baur, B., Wick, H., and Baumgartner, E.R. 1990. Na+-dependent biotin transport into brush-border membrane vesicles from rat kidney. Am.J. Physiol. 258:F840-F847.

32. Spencer, P.D., and Roth, K.S. 1988. On the uptake of biotin by the rat renal tubule. Biochem. Med. Metab. Biol. 40:95-100.

33. Podevin, R.A., and Barbarat, B. 1986. Biotin uptake mechanisms in brush-border and basolateral membrane vesicles isolated from rabbit kidney cortex. Biochim. Biophys. Acta. 856:471-481.

34. Prasad, P.D., Ramamoorthy, S., Leibach, F.H., and Ganapathy, V. 1997. Characterization of a sodium-dependent vitamin transporter mediating the uptake of pantothenate, biotin and lipoate in human placental choriocarcinoma cells. Placenta. 18:527-533.

35. Prasad, P.D., et al. 1998. Cloning and functional expression of a cDNA encoding a mammalian sodium-dependent vitamin transporter mediating the uptake of pantothenate, biotin, and lipoate. J. Biol. Chem. 273:7501-7506.

36. Thoene, J., Baker, H., Yoshino, M., and Sweetman, L. 1981. Biotinresponsive carboxylase deficiency associated with subnormal plasma and urinary biotin. N. Eng. J. Med. 304:817-820.

37. Thoene, J.G., Lemons, R., and Baker, H. 1983. Impaired intestinal absorption of biotin in juvenile multiple carboxylase deficiency. N. Engl. J. Med. 308:639-642.

38. Thoene, J., and Wolf, B. 1983. Biotinidase deficiency in juvenile multiple carboxylase deficiency. Lancet. 13:398. 